\title{
Constrained by Our Connections: White Matter's Key Role in Interindividual Variability in Visual Working Memory Capacity
}

\author{
๑DAli M. Golestani, ${ }^{1}$ Laura Miles, ${ }^{1}$ James Babb, ${ }^{1}$ F. Xavier Castellanos, ${ }^{1,2,3}$ Dolores Malaspina, ${ }^{2,4}$ and ${ }^{\circledR}$ Mariana Lazar $^{1}$ \\ ${ }^{1}$ Department of Radiology, New York University School of Medicine, New York, New York 10016, ${ }^{2}$ Department of Child and Adolescent Psychiatry, New \\ York University School of Medicine, New York, New York 10016, ${ }^{3}$ Nathan Kline Institute for Psychiatric Research, Orangeburg, New York 10962, and \\ ${ }^{4}$ Department of Psychiatry, New York University School of Medicine, New York, New York 10016
}

Visual working memory (VWM) plays an essential role in many perceptual and higher-order cognitive processes. Despite its reliance on a broad network of brain regions, VWM has a capacity limited to a few objects. This capacity varies substantially across individuals and relates closely to measures of overall cognitive function (Luck and Vogel, 2013). The mechanisms underlying these properties are not completely understood, although the amplitude of neural signal oscillations (Vogel and Machizawa, 2004) and brain activation in specific cortical regions (Todd and Marois, 2004) have been implicated. Variability in VWM performance may also reflect variability in white matter structural properties. However, data based primarily on diffusion tensor imaging approaches remain inconclusive. Here, we investigate the relationship between white matter and VWM capacity in human subjects using an advanced diffusion imaging technique, diffusion kurtosis imaging. Diffusion kurtosis imaging provides several novel quantitative white mater metrics, among them the axonal water fraction $\left(f_{\text {axon }}\right)$, an index of axonal density and caliber. Our results show that $59 \%$ of individual variability in VWM capacity may be explained by variations in $f_{\text {axon }}$ within a widely distributed network of white matter tracts. Increased $f_{\text {axon }}$ associates with increased VWM capacity. An additional 12\% in VWM capacity variance may be explained by diffusion properties of the extra-axonal space. These data demonstrate, for the first time, the key role of white matter in limiting VWM capacity in the healthy adult brain and suggest that white matter may represent an important therapeutic target in disorders of impaired VWM and cognition.

Key words: axonal density; diffusion kurtosis imaging; individual differences; microstructure; visual working memory capacity; white matter

\section{Introduction}

Visual working memory (VWM; i.e., the short-term storage of visual information) is supported by a distributed neural system, which in humans includes occipital, prefrontal, parietal, and temporal areas (Ungerleider et al., 1998). Functional imaging has been extensively used to identify the cortical areas involved in VWM (Ungerleider et al., 1998; Zimmer et al., 2010). The relationship between VWM and the white matter pathways interconnecting the VWM network nodes has also been studied primarily using diffusion tensor imaging (DTI). DTI measures, such as fractional anisotropy (FA), quantify water molecules' microscopic diffusion and reflect a variety of microstructural features, among them the degree of myelination and fiber density and

\footnotetext{
Received June 6, 2014; revised Sept. 18, 2014; accepted Sept. 25, 2014.

Author contributions: M.L. designed research; A.M.G., L.M., J.B., F.X.C., D.M., and M.L. performed research; A.M.G. and M.L. analyzed data; A.M.G., F.X.C., and M.L. wrote the paper.

This study was supported by National Institutes of Mental Health Awards R21-MH085228 and R03-MH076180. We thank ResearchMatch for their support with advertising our study.

The authors declare no competing financial interests.

Correspondence should be addressed to Dr. Mariana Lazar, Center for Biomedical Imaging, New York University

School of Medicine, 660 First Avenue, Room 423, New York, NY 10016. E-mail: mariana.lazar@nyumc.org.

DOI:10.1523/JNEUROSCI.2317-14.2014

Copyright $\odot 2014$ the authors $\quad 0270-6474 / 14 / 3414913-06 \$ 15.00 / 0$
}

coherence. Most DTI studies examining the relationship between white matter and working memory (visual or verbal) have focused on pathological groups, such as patients with schizophrenia (Hanlon et al., 2012) or multiple sclerosis (Yu et al., 2012), pathological processes such as aging (Davis et al., 2009), or on early brain development (Klingberg, 2006; Vestergaard et al., 2011; Short et al., 2013; Peters et al., 2014). In healthy adults, modest relationships have been reported between FA and verbal working memory (Karlsgodt et al., 2008; Takeuchi et al., 2011).

A potential limitation of these studies was the use of DTI metrics, which, although highly sensitive to pathological processes, may lack specificity (Walker et al., 2012). Thus, we have used a recently proposed two-compartment model of white matter (Fieremans et al., 2011), based on diffusion kurtosis imaging (DKI), which separates the magnetic resonance signal contribution from intra- and extra-axonal white matter compartments. Several metrics can be obtained using this approach: (1) the axonal water fraction $\left(f_{\text {axon }}\right)$, which describes the volume of the intra-axonal water relative to the total intra- and extra-axonal volume and relates to axonal density and caliber (Barazany et al., 2009; De Santis et al., 2012); (2) the intra-axonal diffusivity $\left(D_{\text {axon }}\right)$, which describes water diffusion within axons, primarily along the axonal axis, and thus reflects microstructural features, 
such as the size, number, and organization of intra-axonal structures; and (3) the extra-axonal axial $\left(A D_{\text {extra }}\right)$ and radial $\left(R D_{\text {extra }}\right)$ diffusivities, which reflect the overall microstructural organization of the extra-axonal space. Recent work has shown that these metrics are sensitive to white matter characteristics that are not described by typical DTI metrics, such as differences in axonal density and caliber in autism (Lazar et al., 2014) and progression from mild cognitive impairment to Alzheimer's disease (Fieremans et al., 2013).

Because kurtosis metrics are more specific to several white matter features that are only indirectly reflected by typical DTI parameters, we hypothesized that they may provide better biomarkers of the relationship between white matter microstructure and physiological markers, such as VWM capacity. Thus, we used a white matter-based voxelwise approach, tract-based spatial statistics (TBSS) (Smith et al., 2006), to investigate correlations between DKI white matter metrics and VWM capacity in a group of 19 healthy male adult participants. The relationship between VWM capacity and DTI-based metrics was also examined.

\section{Materials and Methods}

Participants. Data were acquired from male right-handed healthy controls $30-55$ years of age using a protocol approved by our Institutional Review Board. Our age range was selected to minimize the effects of development or aging. Participants were recruited by advertising in the community and through ResearchMatch and Craigslist websites. All participants provided informed consent and participated in a series of cognitive assessments, including VWM. None of the participants revealed a history of brain disorders or head trauma, a history of alcohol or substance abuse in the past 6 months, or substance-induced psychotic disorder or psychotic disorder due to a general medical condition as determined by DSM-IV criteria. The Structured Clinical Interview for DSM-IV Non-Patient Edition (First et al., 2002) was used to assess the potential presence of psychiatric symptoms. Clinical neurological examination of imaging data, performed as part of the typical workflow in our department, was used to assess the presence of any gross brain abnormalities. Subjects with abnormal imaging findings or those presenting psychiatric symptoms were excluded from the study.

A total of 25 subjects were recruited. Two subjects were excluded because of the presence of depression or autism diagnoses. Three additional subjects were excluded because of brain abnormalities on neuroradiological examination, including abnormal presence of white matter hyperintensities and lesions. One subject participated in the neuropsychological testing but failed to participate in the MRI session. The remaining 19 participants were included in analyses.

The age of the included subjects varied between 31 and 55 years $(42 \pm$ 6 years). Length of education varied between 12 and 19 years ( $15.7 \pm 1.8$ years). Neither education nor the composite global cognitive score (obtained by averaging the $z$-scores for cognitive assessments) and none of the VWM metrics correlated with age $(p>0.80)$.

VWM assessment. VWM capacity was assessed using the Symbol Span subtest of the Wechsler Memory Scale-Fourth Edition (WMS-IV). In Symbol Span, participants are shown a series of abstract symbols on a page for $5 \mathrm{~s}$ and then asked to select these symbols from an array of symbols, in the same order they were presented on the previous page. The number of symbols is increased from trial to trial. Each successful trial receives a 2 point score if symbols are remembered in the correct order and 1 point if all symbols are retrieved, but not in the correct order. The test is terminated after three unsuccessful trials. Symbol Span is a measure of VWM capacity as it reflects the longest list of items that a person can repeat back immediately after presentation.

Image acquisition. Imaging data were acquired on a 3T Trio MRI (Siemens Medical Solutions). Diffusion-weighted images were acquired using Echo Planar Imaging (EPI) for a total of 64 uniformly distributed gradient directions and for two b values $\left(b=1000\right.$ and $\left.2000 \mathrm{~s} / \mathrm{mm}^{2}\right)$. Ten nonweighted diffusion images $\left(b=0 \mathrm{~s} / \mathrm{mm}^{2}\right.$ ) were also collected. A field map image coplanar to the diffusion acquisition was acquired using a pair of non-EPI gradient echo images at two echo times and used to correct for image distortions from B0 field inhomogeneities.

Image processing. Postacquisition processing was performed with locally developed software and the FSL suite (Jenkinson et al., 2012). Data preprocessing included the following: (1) visual inspection of all images for quality assessment with elimination of any artifactual images; (2) coregistration of diffusion images using the FSL Flirt utility; and (3) correction for B0 field inhomogeneities distortion using the acquired field map data and FSL.

After data correction, diffusion and kurtosis tensors were estimated using a constrained linear least square approach (Tabesh et al., 2011). The encoding gradients' matrix was adjusted to compensate for the effect of image rotation from the motion correction step. The diffusion and kurtosis tensors were used to derive $3 \mathrm{D}$ maps of $f_{\text {axon }}, D_{\text {axon }}, A D_{\text {extra }}$ and $R D_{\text {extra }}$. Diffusion tensor data were used to derive the DTI metrics FA and axial (AD) and radial (RD) diffusivity at each brain voxel.

TBSS. Voxelwise analyses were conducted using TBSS (Smith et al., 2006). First, individual FA images of all subjects were aligned to an MNI152 template using nonlinear registration. Then, a mean FA image was created; a threshold of FA $>0.2$ generated an FA skeleton that represents tracts common to all subjects. Individual parametric maps were then projected onto the mean FA skeleton by looking for maximum local values perpendicular to the skeleton. Statistical tests were conducted using the permutation-based nonparametric analysis routine Randomize (Jenkinson et al., 2012) with 5000 iterations. Correlations (positive and negative) were computed to examine the association between each of the diffusion parameters with the Symbol Span Raw Score. As age may influence both microstructural properties and Symbol Span, it was used as a covariate in all analyses to assess relationships between VWM capacity and white matter microstructure independent of potential codependence on age. Primary analyses focused on $f_{\text {axon }}, D_{\text {axon }}, A D_{\text {extra }}$, and $R D_{\text {extra }}$ metrics. Secondary analyses focused on FA, RD, and AD. Correlations were considered significant when $p<0.05$, corrected for multiple comparisons using Threshold Free Cluster Enhancement (Smith and Nichols, 2009).

ROI analyses. ROIs were obtained for the whole white matter and for tract-specific regions. As we were interested in intrinsic tract properties, we chose to focus on white matter regions of high anisotropy as these regions are less affected by fiber crossings and partial volume averaging. Neither DTI nor DKI white matter metrics adequately describes the individual fiber properties in regions where more than one fiber population is present in the same voxel. To account for this limitation, the following approximations were made: (1) regions of high linear anisotropy primarily contain single-fiber populations and thus better reflect intrinsic tract microstructure; (2) white matter skeleton regions are less affected by partial volume averaging with adjacent tracts or gray matter regions; and (3) high anisotropy white matter skeleton regions are representative of the overall intrinsic tract properties. Thus, ROIs were limited to white matter skeleton areas with linear anisotropy metric, $c_{1}$ (Westin et al., 2002) larger than 0.4. Linear anisotropy was selected for determining the threshold in these analyses as it is larger for prolate tensors, which are more likely to reflect single-fiber populations. Tractspecific masks were generated using the Johns Hopkins University tract atlas overlaid onto the white matter skeleton regions with $c_{1}>0.4$. A global white matter mask was also obtained by selecting all skeleton areas that met the same linear anisotropy criterion. DKI and DTI metrics for these ROIs were obtained by averaging all skeleton values covered by the masks.

In a first approach, global- and tract-specific white matter associations with VWM capacity were assessed using two-tailed partial Pearson correlations using age as a covariate. In a second approach, we tested for tract specific associations using two-stage hierarchical multiple linear regression that used Symbol Span as the dependent variable, with age and global white matter value entered as control predictors in the first stage and tract-specific metrics entered as predictors of interest in the second stage. This approach was chosen to identify tract-specific contributions to VWM capacity above global white matter contributions and independent of age. As microstructural properties are highly correlated among various white matter structures (Wahl et al., 2010), only representative 
a

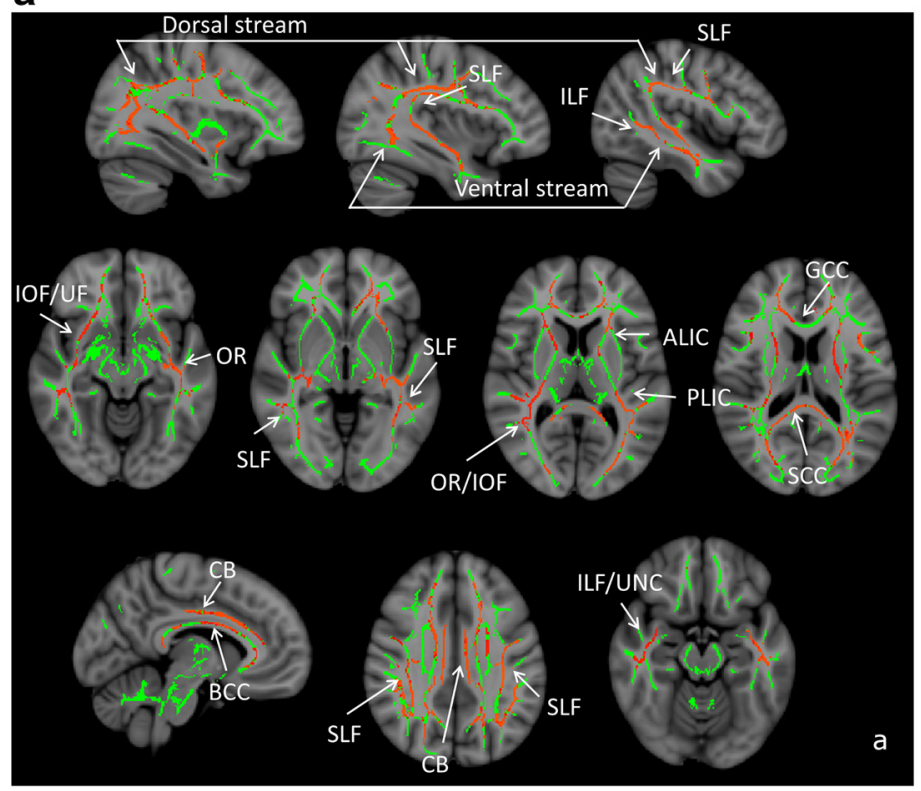

b
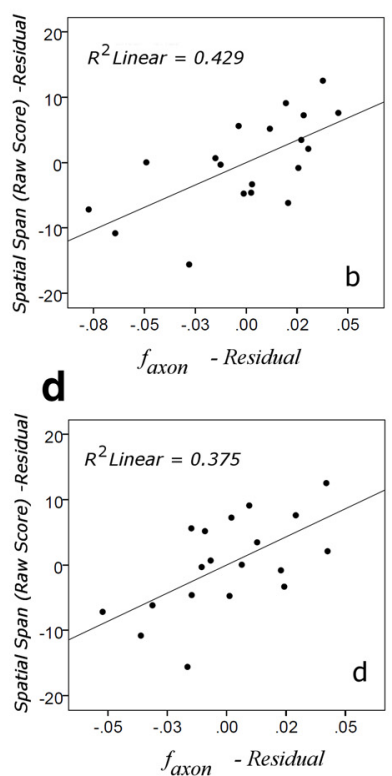

C

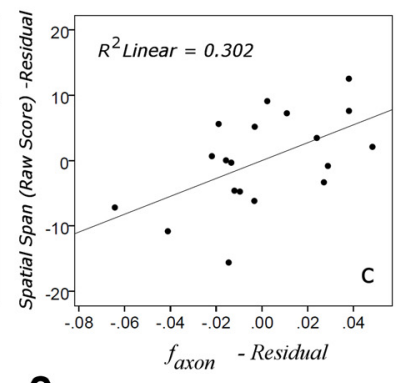

e

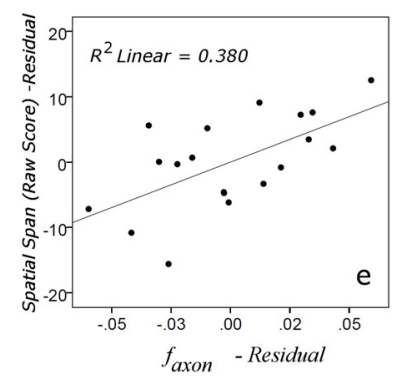

Figure 1. $\boldsymbol{a}$, White matter skeleton regions with $f_{\text {axon }}$ significantly correlated to the Symbol Span Raw Score: the superior longitudinal fasciculus (SLF), inferior longitudinal fasciculus (ILF), cingulum bundle (CB), optic radiation (OR), inferior occipitofrontal fasciculus (IOF), anterior (ALIC) and posterior (PLIC) limbs of the internal capsule, the uncinate fasciculus (UF), and the genu (GCC), body (BCC), and splenium (SCC) of corpus callosum. These areas (orange shades) are overlaid over the white matter skeleton (green) and anatomical T1-weighted brain images. Age was used as a covariate in these analyses. $\boldsymbol{b}-\boldsymbol{e}$, Plots depicting the partial correlations between Symbol Span and mean tract $f_{\text {axon }}$ are shown for the right cingulum bundle ( $\boldsymbol{b}$ ), left inferior occipitofrontal fasciculus (c), left inferior longitudinal fasciculus $(\boldsymbol{d})$, and left superior longitudinal fasciculus $(\boldsymbol{e})$. To control for age, residuals of Symbol Span and mean tract $f_{\text {axon }}$ regression with respect to age are plotted.

tracts were included in the regression analysis to minimize overfitting from highly correlated predictors. Tract selection was performed as follows. First, hierarchical clustering was used to determine groups of tracts with similar properties. Second, forward variable selection was used to identify the best predictors within each group. Hierarchical multiple regression was performed for each of the examined metrics. As different metrics reflect at least in part different white matter properties that may each contribute independently to behavioral performance, a final model was constructed that included contributions from multiple DKI and DTI metrics. Forward selection was used to test whether either global- or tract-specific values of these metrics improved prediction of VWM capacity beyond single metric contributions. Correlations were considered significant if $p<0.01$ to account for multiple comparisons. Statistical analyses were conducted using SPSS Statistics for Windows, Version 21.0 (IBM).

\section{Results}

\section{TBSS analyses}

Voxelwise TBSS results show that VWM capacity positively correlates with $f_{\text {axon }}$ in a wide array of white matter tracts (Fig. 1a). These tracts include two prominent pathways of the ventral and dorsal streams, the inferior and superior longitudinal fasciculi, and the optic radiations and posterior thalamus, which are known to play important roles in visual processes. Additionally, $f_{\text {axon }}$ of major tracts connecting the prefrontal cortex with the occipital, parietal, and temporal lobes (inferior occipitofrontal fasciculi, cingulum bundles, and uncinate fasciculi) was found to correlate to VWM capacity, which may reflect the key role of the prefrontal cortex in VWM processes. Corpus callosum, another structure prominently highlighted in the correlational maps, underlies the integration of visual information across the right and left visual fields. Aside from $f_{\text {axon }}$, none of the other investigated metrics, whether DTI or DKI based, correlated significantly with VWM capacity in any brain region in TBSS analyses after accounting for multiple comparisons.

\section{ROI analyses}

ROI analyses were consistent with the TBSS analyses, with $f_{\text {axon }}$ the only metric correlated with VMW capacity globally $(p<0.05$; Table 1); however, correlations with global $A D_{\text {extra }}$ were also observed at trend level $(p=0.057)$. VWM capacity correlated significantly with $f_{\text {axon }}$ of right cingulum and left superior and inferior longitudinal fasciculi, $D_{\text {axon }}$ of left inferior longitudinal fasciculus, and $A D_{\text {extra }}$ of right cingulum. Scatter plots depicting several tract-specific associations between VWM capacity and $f_{\text {axon }}$ are presented in Figure $1 b-e$. Individual tract correlations of the different DTI and DKI diffusion metrics with VWM capacity are presented in Table 1.

Multiple regression analyses were used to assess the relative contributions of age and global- and tract-specific $f_{\text {axon }}$ values in predicting VWM capacity. The $f_{\text {axon }}$-based hierarchical clustering of the white matter tracts selected four major clusters, with the first three clusters, including the following: (1) left inferior occipitofrontal fasciculus, anterior thalamic radiations, and genu of corpus callosum; (2) right inferior occipitofrontal fasciculus, right and left inferior longitudinal fasciculi, and splenium of the corpus callosum; and (3) superior longitudinal fasciculi and cingulum bundles (superior segment). A fourth cluster included the corticospinal tracts, parahippocampal cingulum bundles, and uncinate fasciculi. The multistep linear regression model identified $f_{\text {axon }}$ values of representative tracts from the first three clusters (the right cingulum, left inferior longitudinal fasciculus, and left inferior occipitofrontal fasciculus) as optimal predictors of VWM capacity controlling for age and global $f_{\text {axon }}$ (Table 2). Among these predictors, the individual tracts were the only ones with significant contributions $(p<0.01)$, with global value not contributing significantly to predicting VWM capacity (Table 
Table 1. Global- and tract-specific associations between Symbol Span Raw Score and diffusion metrics obtained using partial correlations that controlled for age ${ }^{a}$

\begin{tabular}{|c|c|c|c|c|c|c|c|}
\hline Tract & $f_{\text {axon }}$ & $D_{\text {axon }}$ & $R D_{\text {extra }}$ & $A D_{\text {extra }}$ & $F A$ & $R D$ & $A D$ \\
\hline Global white matter & $0.544^{*}(0.019)$ & $0.389(0.111)$ & $0.242(0.334)$ & $0.456(0.057)$ & $0.192(0.446)$ & $-0.155(0.540)$ & $0.064(0.801)$ \\
\hline Left cingulum & $0.542^{*}(0.020)$ & $0.385(0.115)$ & $0.264(0.290)$ & $0.555^{*}(0.017)$ & $0.362(0.139)$ & $-0.211(0.400)$ & $0.511^{*}(0.030)$ \\
\hline Right cingulum & $0.655^{* *}(0.003)$ & $0.501^{*}(0.034)$ & $0.198(0.432)$ & $0.611^{* *}(0.007)$ & $0.470^{*}(0.049)$ & $-0.299(0.229)$ & $0.461(0.054)$ \\
\hline Splenium of corpus callosum & $0.538^{*}(0.021)$ & $0.478^{*}(0.045)$ & $0.127(0.615)$ & $0.374(0.126)$ & $0.161(0.522)$ & $-0.095(0.709)$ & $0.141(0.577)$ \\
\hline Genu of corpus callosum & $0.347(0.158)$ & $0.368(0.133)$ & $0.041(0.872)$ & $0.189(0.453)$ & $0.082(0.748)$ & $-0.125(0.620)$ & $-0.094(0.710)$ \\
\hline Left inferior longitudinal fasciculus & $0.613^{* *}(0.007)$ & $0.594^{* *}(0.009)$ & $0.302(0.223)$ & $0.474^{*}(0.047)$ & $0.249(0.319)$ & $-0.062(0.805)$ & $0.293(0.238)$ \\
\hline Left inferior occipitofrontal fasciculus & $0.559^{*}(0.018)$ & $0.477^{*}(0.045)$ & $0.326(0.187)$ & $0.456(0.057)$ & $0.137(0.589)$ & $-0.013(0.960)$ & $0.293(0.239)$ \\
\hline Left superior longitudinal fasciculus & $0.616^{* *}(0.006)$ & $0.471^{*}(0.049)$ & $0.326(0.187)$ & $0.379(0.121)$ & $0.184(0.464)$ & $-0.173(0.493)$ & $0.048(0.850)$ \\
\hline Right superior longitudinal fasciculus & $0.484^{*}(0.042)$ & $0.224(0.371)$ & $-0.018(0.944)$ & $0.174(0.490)$ & $0.225(0.369)$ & $-0.308(0.214)$ & $-0.113(0.656)$ \\
\hline Left uncinate fasciculus & $0.412(0.089)$ & $0.426(0.078)$ & $0.322(0.193)$ & $0.339(0.168)$ & $0.136(0.589)$ & $-0.028(0.911)$ & $0.476^{*}(0.046)$ \\
\hline
\end{tabular}

${ }^{a} p$ values are listed in parentheses.

${ }^{*} p<0.05 ;{ }^{* *} p<0.01$ (significant correlations)

Table 2. Regression models describing VWM capacity dependence on age, globaland tract-specific $f_{\text {axon, }}$ and global $A D_{\text {extra }}$

\begin{tabular}{|c|c|c|c|c|}
\hline Model & Predictors & $\begin{array}{l}\text { Significance } \\
\text { (predictors) }\end{array}$ & $\begin{array}{l}\text { Significance } \\
\text { (full model) }\end{array}$ & $\begin{array}{l}\text { Adjusted } \mathrm{R}^{2} \\
\text { (full model) }\end{array}$ \\
\hline \multirow[t]{2}{*}{1} & Age & 0.567 & \multirow[t]{2}{*}{0.046} & \multirow[t]{2}{*}{0.234} \\
\hline & $f_{a x o n}$ (global) & 0.019 & & \\
\hline \multirow[t]{5}{*}{2} & Age & 0.061 & \multirow[t]{5}{*}{0.005} & \multirow[t]{5}{*}{0.565} \\
\hline & $f_{a x o n}$ (global) & 0.732 & & \\
\hline & $f_{\text {axon }}$ (right cingulum) & 0.004 & & \\
\hline & $\begin{array}{l}f_{\text {axon }} \text { (left inferior longi- } \\
\text { tudinal fasciculus) }\end{array}$ & 0.042 & & \\
\hline & $\begin{array}{l}f_{\text {axon }} \text { (left inferior fronto- } \\
\text { occipital fasciculus) }\end{array}$ & 0.008 & & \\
\hline \multirow[t]{4}{*}{3} & Age & 0.004 & \multirow[t]{4}{*}{0.002} & \multirow[t]{4}{*}{0.592} \\
\hline & $f_{\text {axon }}$ (right cingulum) & 0.000 & & \\
\hline & $\begin{array}{l}f \text { (left inferior longitudi- } \\
\text { nal fasciculus) }\end{array}$ & 0.001 & & \\
\hline & $\begin{array}{l}f_{\text {axon }} \text { (left inferior fronto- } \\
\text { occipital fasciculus) }\end{array}$ & 0.001 & & \\
\hline \multirow[t]{5}{*}{4} & Age & 0.004 & \multirow[t]{5}{*}{0.000} & \multirow[t]{5}{*}{0.718} \\
\hline & $f_{\text {axon }}$ (right cingulum) & 0.000 & & \\
\hline & $\begin{array}{l}f_{\text {axon }} \text { (left inferior longi- } \\
\text { tudinal fasciculus) }\end{array}$ & 0.001 & & \\
\hline & $\begin{array}{l}f_{\text {axon }} \text { (left inferior fronto- } \\
\text { occipital fasciculus) }\end{array}$ & 0.001 & & \\
\hline & $A D_{\text {extra }}$ (global) & 0.018 & & \\
\hline
\end{tabular}

2) when the individual tracts were included in the model. The age and global value model explained only $23 \%$ of the individual variability in the VWM capacity. The full model accounted for $57 \%$ of the interindividual variation in performance. Removal of global $f_{\text {axon }}$, a nonsignificant predictor, increased the adjusted $R^{2}$ of the overall model to $59 \%$.

Hierarchical regression was also used to assess whether accounting for combined tract contributions in addition to global contributions improves prediction of VWM capacity for metrics other than $f_{\text {axon }}$. No significant relationships ( $p>$ 0.01 ) were found for any of the metrics, whether for models including only age and global values as predictors or more complex models that also included representative tract contributions. Representative results are presented for FA in Table 3.

Evaluation of improvement in the $f_{\text {axon }}$ model by considering additional white matter microstructural features described by the other DTI and DKI metrics showed that global $A D_{\text {extra }}$ further improved the predictive value of the model (Table 2). The combined $f_{\text {axon }}-A D_{\text {extra }}$ model explained $72 \%$ of the variance in individual VWM capacity.
Table 3. Regression models describing VWM capacity dependence on age and global- and tract-specific $\mathrm{FA}^{a}$

\begin{tabular}{lllll}
\hline Model & Predictors & $\begin{array}{l}\text { Significance } \\
\text { (predictors) }\end{array}$ & $\begin{array}{l}\text { Significance } \\
\text { (full model) }\end{array}$ & $\begin{array}{l}\text { Adjusted R } \\
\text { (full model) }\end{array}$ \\
\hline 1 & Age & 0.562 & 0.571 & -0.049 \\
& FA (global) & 0.446 & & \\
2 & Age & 0.628 & 0.174 & 0.130 \\
& FA (global) & 0.449 & & \\
& FA (right cingulum) & 0.056 & & \\
& ${ }^{a}$ No significant relationships were found using either global- or tract-specific FA.
\end{tabular}

\section{Discussion}

VWM capacity has been modeled in terms of the ability to link distinct cell assemblies across multiple cortical areas (Luck and Vogel, 2013). Our results strongly suggest that the communication routes among cortical areas (i.e., white matter pathways) are a key factor in determining individual VWM capacity. As $f_{\text {axon }}$ reflects axonal density and caliber, its relationship to VWM capacity suggests that individuals with denser white matter pathways or larger axons may be able to maintain a larger amount of information "online" for use by various network processors. From this perspective, $f_{\text {axon }}$ may index white matter information processing capacity. The rate of information exchange depends on axonal firing rate, which appears to be linearly related to axonal caliber (Perge et al., 2012). Thus, larger axons may contribute to faster information exchange between different network processors and increase the range of synchronized oscillations among distinguishable cell assemblies representing distinct objects stored in memory. Faster axons may also be more proficient in supporting the recurrent feedback loops that are assumed by some models to subserve VWM representations (Luck and Vogel, 2013).

The TBSS analyses delineate the extended network involved in VWM processes. Tract-specific analyses further show that tracts connecting prefrontal, occipital, parietal, and temporal lobe are significant predictors of VWM capacity, contributing beyond global white matter axon properties and independent of age. Thus, these data suggest that VWM storage and retrieval involve collaboration among multiple nodes of the VWM network; the size and density of the axons linking these nodes appear to play an essential role in determining VWM capacity. An additional, but more modest, association was found between VWM capacity and global $A D_{\text {extra }}$. This finding suggests that organization and microarchitecture of extra-axonal space, which include glial cells, such as astrocytes and oligodendrocytes as well as extracellular space, may play a role in transmission of information by white matter. Glia and extracellular space have been long thought to contribute 
to information transmission in the brain (Fields and StevensGraham, 2002). The exact nature of the involved biological substrates and associated diffusion mechanisms, however, is yet to be determined.

Overall, the white matter microstructural features evaluated in this study appear to explain a substantial proportion of the individual variability in VWM capacity (72\%). The remaining variance may be explained by features not described by the parameters we measured, such as contributions from gray matter, or differences in overall network organization.

One of the most intriguing properties of VWM capacity is its yet to be explained strong correlation with global cognitive performance (Luck and Vogel, 2013). The extended network of tracts we found related to VWM capacity performance are most likely also involved in more complex cognitive functions. Accordingly, we propose that reliance on a similar white matter network explains, at least in part, the high correlation between VWM capacity and measures of overall cognitive function (Luck and Vogel, 2013). Thus, white matter may represent a promising therapeutic target in disorders of both impaired VWM and impaired general cognitive abilities.

We found no significant correlations between DTI metrics and VWM capacity in our sample. This is consistent with earlier work that found no correlations between FA and cognitive metrics, including VWM capacity, in adulthood (Tamnes et al., 2011; Peters et al., 2014), although modest relationships with verbal working memory have been reported (Takeuchi et al., 2011), sometimes using only analyses restricted to one or two white matter tracts (Karlsgodt et al., 2008). Of note here is that FA correlations with various aspects of working memory (verbal or spatial) have been more widely reported in pathology, such as schizophrenia or multiple sclerosis. Such disorders have wellknown impairments in FA; thus, it is expected that working memory in those cases may have different neural mechanisms than in typical healthy brain, with impaired features playing a role in modulating performance.

The DKI-based metrics used in this study are obtained assuming a two-compartment model of white matter microstructure. However, the kurtosis tensor also allows derivation of several model-independent parameters, including mean $(\mathrm{MK})$, radial (RK), and axial kurtosis (AK) (Jensen and Helpern, 2010). RK was shown to be highly correlated with $f_{\text {axon }}$ (De Santis et al., 2012). Although we focused our main analyses on the intra- and extra-axonal diffusion metrics due to their relevance to specific white matter features, additional investigation of the relationship with RK in our data revealed, as expected, significant correlations with VWM capacity in the same regions as $f_{\text {axon }}$ (data not shown). Thus, the relationships between VWM capacity and white matter microstructural properties are model-independent.

In conclusion, we found that white matter represents an important anatomical substrate of VWM capacity interindividual variability in the adult healthy brain. The DKI-based metric, $f_{\text {axon }}$, was found to be a strong noninvasive imaging predictor of individual visual memory capacity.

\section{References}

Barazany D, Basser PJ, Assaf Y (2009) In vivo measurement of axon diameter distribution in the corpus callosum of rat brain. Brain 132:12101220. CrossRef Medline

Davis SW, Dennis NA, Buchler NG, White LE, Madden DJ, Cabeza R (2009) Assessing the effects of age on long white matter tracts using diffusion tensor tractography. Neuroimage 46:530-541. CrossRef Medline

De Santis S, Assaf Y, Jones DK (2012) Using the biophysical CHARMED model to elucidate the underpinnings of contrast in diffusional kurtosis analysis of diffusion-weighted MRI. MAGMA 25:267-276. CrossRef Medline

Fields RD, Stevens-Graham B (2002) New insights into neuron-glia communication. Science 298:556-562. CrossRef Medline

Fieremans E, Jensen JH, Helpern JA (2011) White matter characterization with diffusional kurtosis imaging. Neuroimage 58:177-188. CrossRef Medline

Fieremans E, Benitez A, Jensen JH, Falangola MF, Tabesh A, Deardorff RL, Spampinato MV, Babb JS, Novikov DS, Ferris SH, Helpern JA (2013) Novel white matter tract integrity metrics sensitive to Alzheimer disease progression. AJNR Am J Neuroradiol 34:2105-2112. CrossRef Medline

First MB, Spitzer RL, Gibbon M, Williams JBW (2002) Structured clinical interview for DSM-IV-TR Axis I disorders, Research Version, NonPatient Edition. (SCID-I/NP). In: Biometrics research. New York: New York State Psychiatric Institute.

Hanlon FM, Houck JM, Klimaj SD, Caprihan A, Mayer AR, Weisend MP, Bustillo JR, Hamilton DA, Tesche CD (2012) Frontotemporal anatomical connectivity and working-relational memory performance predict everyday functioning in schizophrenia. Psychophysiology 49:1340-1352. CrossRef Medline

Jenkinson M, Beckmann CF, Behrens TE, Woolrich MW, Smith SM (2012) FSL. Neuroimage 62:782-790. CrossRef Medline

Jensen JH, Helpern JA (2010) MRI quantification of non-Gaussian water diffusion by kurtosis analysis. NMR Biomed 23:698-710. CrossRef Medline

Karlsgodt KH, van Erp TG, Poldrack RA, Bearden CE, Nuechterlein KH, Cannon TD (2008) Diffusion tensor imaging of the superior longitudinal fasciculus and working memory in recent-onset schizophrenia. Biol Psychiatry 63:512-518. CrossRef Medline

Klingberg T (2006) Development of a superior frontal-intraparietal network for visuo-spatial working memory. Neuropsychologia 44:21712177. CrossRef Medline

Lazar M, Miles LM, Babb JS, Donaldson JB (2014) Axonal deficits in young adults with high functioning autism and their impact on processing speed. Neuroimage Clin 4:417-425. CrossRef Medline

Luck SJ, Vogel EK (2013) Visual working memory capacity: from psychophysics and neurobiology to individual differences. Trends Cogn Sci 17: 391-400. CrossRef Medline

Perge JA, Niven JE, Mugnaini E, Balasubramanian V, Sterling P (2012) Why do axons differ in caliber? J Neurosci 32:626-638. CrossRef Medline

Peters BD, Ikuta T, DeRosse P, John M, Burdick KE, Gruner P, Prendergast DM, Szeszko PR, Malhotra AK (2014) Age-related differences in white matter tract microstructure are associated with cognitive performance from childhood to adulthood. Biol Psychiatry 75:248-256. CrossRef Medline

Short SJ, Elison JT, Goldman BD, Styner M, Gu H, Connelly M, Maltbie E, Woolson S, Lin W, Gerig G, Reznick JS, Gilmore JH (2013) Associations between white matter microstructure and infants' working memory. Neuroimage 64:156-166. CrossRef Medline

Smith SM, Nichols TE (2009) Threshold-free cluster enhancement: addressing problems of smoothing, threshold dependence and localisation in cluster inference. Neuroimage 44:83-98. CrossRef Medline

Smith SM, Jenkinson M, Johansen-Berg H, Rueckert D, Nichols TE, Mackay CE, Watkins KE, Ciccarelli O, Cader MZ, Matthews PM, Behrens TE (2006) Tract-based spatial statistics: voxelwise analysis of multi-subject diffusion data. Neuroimage 31:1487-1505. CrossRef Medline

Tabesh A, Jensen JH, Ardekani BA, Helpern JA (2011) Estimation of tensors and tensor-derived measures in diffusional kurtosis imaging. Magn Reson Med 65:823-836. CrossRef Medline

Takeuchi H, Taki Y, Sassa Y, Hashizume H, Sekiguchi A, Fukushima A, Kawashima R (2011) Verbal working memory performance correlates with regional white matter structures in the frontoparietal regions. Neuropsychologia 49:3466-3473. CrossRef Medline

Tamnes CK, Fjell AM, Østby Y, Westlye LT, Due-Tønnessen P, Bjornerud A, Walhovd KB (2011) The brain dynamics of intellectual development: waxing and waning white and gray matter. Neuropsychologia 49:36053611. CrossRef Medline

Todd JJ, Marois R (2004) Capacity limit of visual short-term memory in human posterior parietal cortex. Nature 428:751-754. CrossRef Medline Ungerleider LG, Courtney SM, Haxby JV (1998) A neural system for human 
visual working memory. Proc Natl Acad Sci U S A 95:883-890. CrossRef Medline

Vestergaard M, Madsen KS, Baaré WF, Skimminge A, Ejersbo LR, Ramsøy TZ, Gerlach C, Akeson P, Paulson OB, Jernigan TL (2011) White matter microstructure in superior longitudinal fasciculus associated with spatial working memory performance in children. J Cogn Neurosci 23:21352146. CrossRef Medline

Vogel EK, Machizawa MG (2004) Neural activity predicts individual differences in visual working memory capacity. Nature 428:748-751. CrossRef Medline

Wahl M, Li YO, Ng J, Lahue SC, Cooper SR, Sherr EH, Mukherjee P (2010) Microstructural correlations of white matter tracts in the human brain. Neuroimage 51:531-541. CrossRef Medline
Walker L, Gozzi M, Lenroot R, Thurm A, Behseta B, Swedo S, Pierpaoli C (2012) Diffusion tensor imaging in young children with autism: biological effects and potential confounds. Biol Psychiatry 72:1043-1051. CrossRef Medline

Westin CF, Maier SE, Mamata H, Nabavi A, Jolesz FA, Kikinis R (2002) Processing and visualization for diffusion tensor MRI. Med Image Anal 6:93-108. CrossRef Medline

Yu HJ, Christodoulou C, Bhise V, Greenblatt D, Patel Y, Serafin D, MaleticSavatic M, Krupp LB, Wagshul ME (2012) Multiple white matter tract abnormalities underlie cognitive impairment in RRMS. Neuroimage 59: 3713-3722. CrossRef Medline

Zimmer HD, Münzer S, Umla-Runge K (2010) Visuo-spatial working memory as a limited resource of cognitive processing. Berlin: Springer. 